\title{
SENNA MACRANTHERA (LEGUMINOSAE), UNA ESPECIE ORNAMENTAL NATURALIZADA EN LA ARGENTINA
}

\author{
HÉCTOR A. KELLER ${ }^{1}$, JULIO A. HURRELL², RICARDO O. VANNI ${ }^{1}$ \& GUSTAVO DELUCCHI ${ }^{3}$
}

\begin{abstract}
Summary: Keller, H. A., J. A. Hurrell, R. O. Vanni \& G. Delucchi. 2012. Senna macranthera (Leguminosae), an ornamental species naturalized in Argentina. Bonplandia 21(1): 55-60.

This paper includes the first report of Senna macranthera (DC. ex Collad.) H. S. Irwin \& Barneby (Leguminosae) as naturalized species in the province of Misiones, Argentina. Its description, distribution, phenology, uses, comments about its naturalization and reference material are presented.
\end{abstract}

Key words: Legumes, flora of Misiones, Argentina, ornamentals, naturalization.

\begin{abstract}
Resumen: Keller, H. A., J. A. Hurrell, R. O. Vanni \& G. Delucchi. 2012. Senna macranthera (Leguminosae), una especie ornamental naturalizada en la Argentina. Bonplandia 21(1): 55-60.

Este trabajo incluye el primer registro de Senna macranthera (DC. ex Collad.) H. S. Irwin \& Barneby (Leguminosae) como especie naturalizada en la provincia de Misiones, Argentina, su descripción, distribución, fenología, utilidades, comentarios sobre su naturalización y material de referencia.
\end{abstract}

Palabras clave: Leguminosas, flora de Misiones, Argentina, ornamentales, naturalización.

\section{Introducción}

El género Senna Mill. pertenece a la familia Leguminosae, subfam. Caesalpinioideae, tribu Cassieae, subtribu Cassiinae. Comprende entre 295 y 300 especies, principalmente americanas, con buena representación en África, Madagascar y Australia, y escasa en el Sudeste asiático y Oceanía. En su mayoría son especies tropicales, pocas se extienden a zonas templadas y, por excepción, a zonas frías de ambos hemisferios (Irwin \& Barneby, 1981, 1982; Marazzi et al., 2006; Ulibarri, 2008).
En Sudamérica se encuentran ca. 214 especies (Ulibarri, 2008), de las cuales unas 30 crecen en la Argentina (Instituto de Botánica Darwinion, 2012). Una de ellas, S. alata (L.) Roxb. (= Cassia alata L.), arbusto probablemente nativo de Sudamérica septentrional, naturalizada desde los Estados Unidos hasta la Argentina, ha sido citada en nuestro país para Formosa, Chaco, Misiones y Corrientes (Instituto de Botánica Darwinion, 2012; Irwin \& Barneby, 1982).

Muchas especies del género se destacan por su valor ornamental, en especial por su abundante y llamativa floración, siendo frecuente su

${ }^{1}$ Instituto de Botánica del Nordeste, Casilla de Correo 209, 3400-Corrientes, Argentina. E-mail: hakeller2000@ yahoo.com.ar

${ }^{2}$ LEBA. Facultad de Ciencias Naturales y Museo, Universidad Nacional de La Plata, Calle 64 nro. 3, 1900-La Plata, Argentina. Investigador CONICET. E-mail: juliohurrell@gmail.com

${ }^{3}$ Facultad de Ciencias Naturales y Museo, Universidad Nacional de La Plata, Paseo del Bosque s.nro., 1900-La Plata, Argentina. E-mail: delucchi@fcnym.unlp.edu.ar 
cultivo en el arbolado urbano y como elemento decorativo de parques y jardines. Entre otras, las nativas de la Argentina: S. corymbosa (Lam.) H. S. Irwin \& Barneby (= Cassia corymbosa Lam.), "sen del campo", S. spectabilis (DC.) H. S. Irwin \& Barneby (=C. spectabilis DC.), "carnaval", S. pendula (Humb. \& Bonpl. ex Willd.) H. S. Irwin \& Barneby $(=C$. pendula Humb. \& Bonpl. ex Willd.), "pito canuto", y la africana S. didymobotrya (Fresen.) H. S. Irwin \& Barneby (=C. didymobotrya Fresen.), "vainillo africano". También son importantes algunas especies medicinales, como S. alexandrina Mill., "sen", cuyas hojas y frutos se comercializan en herboristerías de nuestro país (Burkart, 1952, 1978; Dimitri \& Rial Alberti, 1954; Hurrell et al., 2004, 2011c,d; Lorenzi, 1992; Ulibarri, 2008; Ulibarri et al., 2006).

En Misiones, el género Senna se halla representado por 10 especies: 9 nativas y la naturalizada $S$. alata (Instituto de Botánica Darwinion, 2012). A estas se agrega aquí $S$. macranthera (DC. ex Collad.) H. S. Irwin \& Barneby, otra especie naturalizada que se cita por primera vez para nuestro país, sobre la base de ejemplares coleccionados en la provincia, en los departamentos de San Pedro, Libertador General San Martín, San Ignacio y Cainguás. Esta se suma a las 69 especies de Leguminosas adventicias presentes en la Argentina (Delucchi et al., 2011).

\section{Resultados}

Senna macranthera (DC. ex Collad.) H.S. Irwin \& Barneby, Mem. New York Bot. Gard. 35 (1): 181. 1982.

Cassia macranthera DC. ex Collad., Hist. Nat. Méd. Casses 99, tab. 8. 1816.

Cassia speciosa Schrad., Gött. Gel. Anz. 1: 718. 1821.

Iconografia: Colladon, loc. cit.; Rodrigues et al., 2005: 8, fig. 12.

Árboles de 3-10 (-15) m alt.; ramas pubescentes o glabrescentes. Estípulas linear-lanceoladas a setiformes, 7-10 mm long., caedizas. Hojas paripinnadas, 12-23 cm long.; pecíolo 2-5,2 cm long.; folíolos 2 pares $(4,8) 5,8-11(-12,7) \times(-2,8)$ de $3,5-4,8$ $\mathrm{cm}$, opuestos, obovados, base oblicua, ápice agudo, epifilo glabrescente, hipofilo finamente pubescente; glándulas nectaríferas ovoides, globosas o fusiformes, sésiles o estipitadas, presentes entre los folíolos del primer par, menos frecuentemente entre los del segundo par; estipela caediza, $0,4 \mathrm{~cm}$ long., junto a la glándula distal. Racimos axilares y/o panículas terminales, 7-20 flores por racimo; pedúnculo (8-)15-40 mm long.; brácteas oval-lanceoladas, 2-4 mm long., persistentes; pedicelos 15-43 $\mathrm{mm}$ long. Flores perfectas, zigomorfas. Sépalos imbricados, 3-7 mm long., pubescentes. Pétalos, amarillos, el centro-adaxial de 3,6-3,8 x 2,3-2,5 $\mathrm{cm}$, los latero-adaxiales y abaxiales de 3,2$3,6 \times 1,8-2,2 \mathrm{~cm}$, base aguda, ápice obtuso. Estambres medianos 4, con filamentos 1-4 $\mathrm{mm}$ long. y anteras 5-9 $\mathrm{mm}$ long.; estambres abaxiales 3 , con anteras isomórficas, 1 central con filamento 2-4 $\mathrm{mm}$ long. y antera 8-11 $\mathrm{mm}$ long., 2 laterales con filamentos 2-5 mm long. y anteras 7-12 mm long. Gineceo 17-25 mm long.; ovario recto, pluriovulado; estilo incurvo. Legumbres cilíndricas, péndulas, estipitadas, rectas, $15,5-21 \mathrm{~cm}$ long. $x$ 1,4-2 cm lat., glabras, tardíamente dehiscentes, epicarpo transversalmente corrugado, pubérulo, pulpa fétida, endocarpo seco, tardíamente dehiscentes. Semillas 2-seriadas, obcordiformes, 7-8 $\mathrm{mm}$ long. x 5,8-6,5 mm lat., castaño oscuras, brillantes. $x=13$ (Biondo et al., 2005).

Distribución geográfica y ecología: Especie variable nativa de Venezuela, Ecuador, Perú y Brasil. En este último país se distribuye desde Piauí hasta San Pablo, principalmente en la mata atlántica, también en la Caatinga y el Cerrado; y se cultiva y crece adventicia entre San Pablo y Rio Grande do Sul (Instituto de Botánica Darwinion, 2012; Irwin \& Barneby, 1982; Jørgensen \& León-Yánez, 1999; Oberlaender, 2006; Rodrigues et al., 2005; Souza \& Bortoluzzi, 2010).

En Paraguay, crece escapada de cultivo en los departamentos de Caaguazú, Canindeyú y San Pedro (Marazzi et al., 2006). En algunos sitios de Brasil es invasora en comunidades naturales, áreas cultivadas y urbanas (Biondi \& Pedrosa-Macedo, 2008; Blum et al., 2008; Kiill et al., 2000).

Fenología: Florece en abundancia desde principios del verano hasta mediados del otoño. 
Fructifica en otoño e invierno. La polinización es entomófila. La dispersión es zoocora, en especial, mediante aves frugívoras (Irwin \& Barneby, 1982; Oberlaender, 2006).

Nombres vernáculos: En la provincia de Misiones, quienes la cultivan le dan las siguientes denominaciones: "lluvia de oro", por su floración abundante; "pata de vaca" por sus foliolos distales que se asemejan a los de especies de las especies de Bauhinia L.; "casia", es un nombre que se aplica por quienes la cultivan, debido a su anterior pertenencia al género Cassia L.

Los guaraníes Mbya la denominan "kaperyva okáre" ("kape": café; "ryva": fruto; "oka": patio, jardín; "re": de). Es decir "café de los patios", por su similitud con congéneres llamados "kaperyva" (fruto del café), cuyas semillas son utilizadas para la preparación de bebidas similares al café (Gonzáles Torres, 1996; Marazzi et al., 2006). Esta denominación híbrida (kape: 'café'; yva: 'fruto'), enunciada y transcripta como "taperyva", ha dado lugar a interpretaciones etimológicas equívocas tales como "fruto del pueblo abandonado" (Cadogan, 1957; Marazzi et al., 2006).

En Brasil, recibe los nombres: "manduirana", "bolão de-ouro", "chuva-de-ouro", "fedegoso", "pau-de-ovelha" (Rodrigues et al., 2005), "aleluia" (Branco et al., 2011), "são João" (Kiill et al., 2000), "cabo verde", "ibixuna", "mamangá", "pau fava", "tararaçu” (Lorenzi, 1992).

Usos: Se cultiva en especial por su valor ornamental, para el arbolado urbano, parques y jardines; se ha introducido en diversos países. Las hojas presentan un elevado tenor de nitrógeno y los ejemplares son buenos productores de hojarasca, por lo que tiene importancia como abono orgánico de sistemas agroforestales (Jaramillo-Botero et al., 2008). Asimismo, presenta muy buena respuesta a la fertilización química, por lo que puede utilizarse en la recomposición de áreas degradadas (Cruz et al., 2010). Es una planta melífera. La madera se emplea para leña y carbón, y para confeccionar juguetes y cajas livianas (Lorenzi, 1992).

En Brasil, se ha empleado en medicina tradicional como remedio antiséptico (Diaz et al., 2010) y antisifilítico (Mors et al., 2000). Se ha estudiado la actividad antimicrobiana, leishmanicida, antioxidante, antiinflamatoria, laxante y sobre la motilidad intestinal de los extractos de las hojas. Éstas contienen cumarinas, saponinas, taninos, flavonoides, compuestos fenólicos, triterpenoides, antronas y esteroides (Diaz et al., 2010; Guarize, 2009; Guarize et al., 2011). Se han aislado antraquinonas de la corteza (Branco et al., 2011) y de las raíces (Mendonça et al., 2011), utilizadas en la elaboración de pigmentos y medicamentos. Del endosperma de las semillas se han obtenido compuestos con actividad anticoagulante (Pires et al., 2001).

Proceso de naturalización: En la provincia de Misiones, se ha indicado su introducción con fines ornamentales hace varias décadas (Dimitri \& Rial Alberti, 1954; Burkart, 1978). La diseminación zoocora habría favorecido su situación inicial como especie escapada de cultivo. En la actualidad, crece naturalizada en ambientes alterados, tales como selvas secundarias, bordes de caminos (Fig. 1A), bajo el dosel de monocultivos forestales y también en bordes de selva primaria, donde alcanza alturas de hasta $15 \mathrm{~m}$ (Fig. 1B). La mayoría de los ejemplares adultos que crecen en estos ambientes son de tamaño mediano (5-10 m), pero también se ha observado una prolífica regeneración natural en capueras o sucesiones secundarias cercanas a áreas urbanas donde se la cultiva abundantemente.

El término "naturalizada" se refiere a especies exóticas que se escapan de cultivo y, luego, se dispersan y expanden por sus propios medios, se establecen e integran a la flora local sin intervención humana. Los elementos naturalizados pueden devenir "invasores", si su expansión es amplia y compromete tanto la biodiversidad como la funcionalidad de las comunidades locales (Pysek et al., 2004; Pysek \& Richardson, 2006; Richardson et al., 2000). Este tema de interés central en las investigaciones sobre las invasiones biológicas en el mundo, en especial, en relación a las especies introducidas con fines ornamentales (Dehnen-Schmutz et al., 2007; Dehnen-Schmutz \& Touza, 2008; Reichard \& White. 2001).

El primer registro de una especie naturalizada 

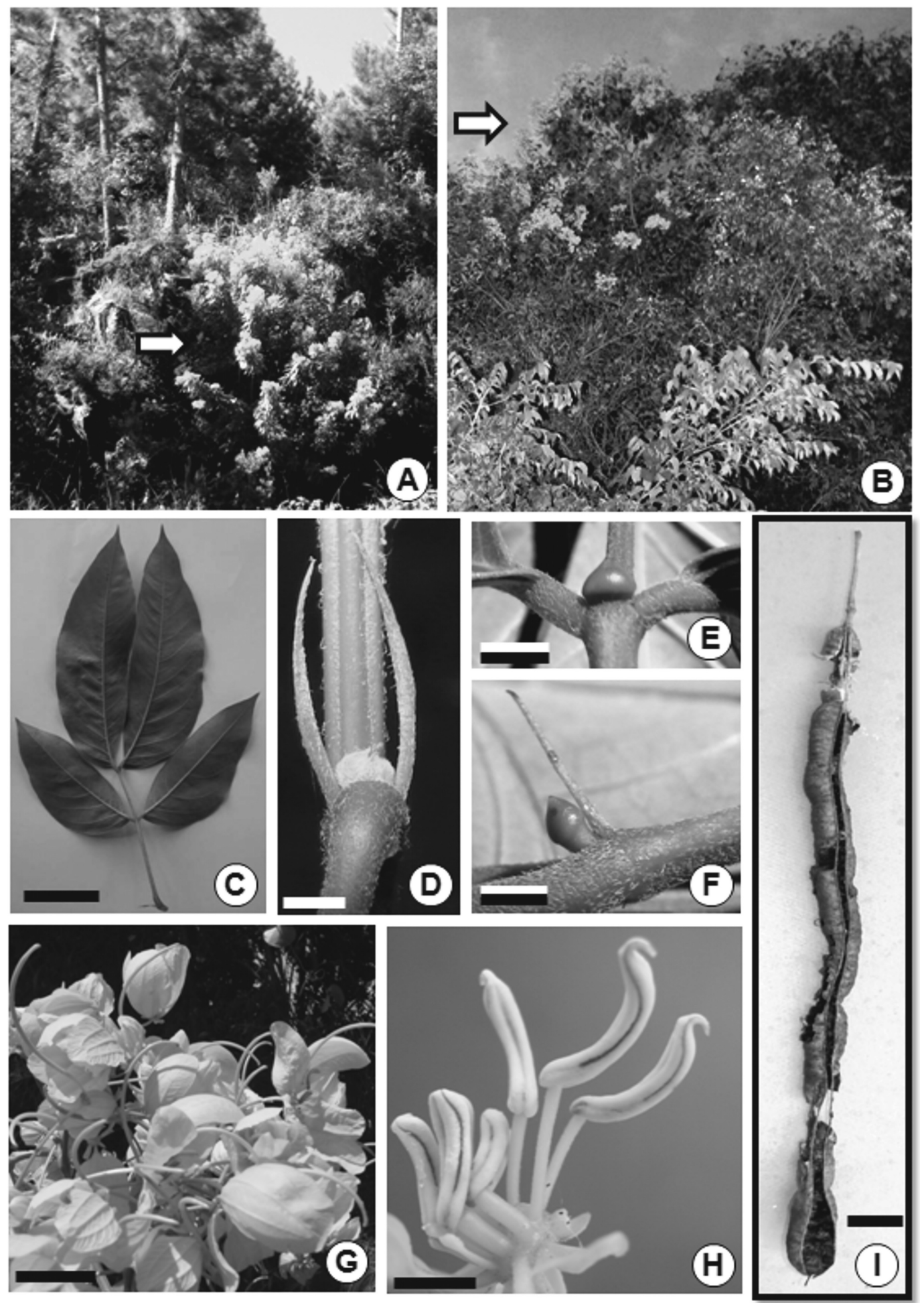

Fig. 1. Senna macranthera. A: Ejemplar en borde de monocultivo de pino. B: Ejemplar en borde de selva. C: Nomófilo mostrando el hipofilo. D: Tallo, yema y estípulas. E: Glándula entre el primer par de folíolos. F: Glándula y estipela entre el segundo par de folíolos. G: Flores. H: Estambres. I: Fruto seco. Escalas en cm: C: 4; D: 0,2; E yF: 0,15; G: 3; H: 4; I: 1,5. A: Keller \& Paredes 10017; B-I: Keller \& Paredes 10041. 
es relevante para analizar su comportamiento futuro dentro del proceso de naturalización, es decir, para evaluar si la especie puede convertirse en invasora. En el caso de $S$. macranthera, este primer registro es significativo, dado que ha sido señalada como invasora en Brasil.

De este modo, la presente contribución, al igual que otros trabajos anteriores en la misma línea (Delucchi et al., 2011; Delucchi \& Hurrell, 2011; Delucchi \& Keller, 2010; Hurrell et al., 2010, 2011a,b; Keller et al., 2011), aporta al estudio de las especies potencialmente invasoras presentes en nuestro país.

Material examinado: ARGENTINA. Misiones. San Pedro. Paraje Gramado, ruta prov. 20, $26^{\circ} 31^{\prime}$ 49,9" S - 5406'05,5" W; 19-III-2011, fl., Keller \& Paredes 10017 (CTES); Libertador Gral. San Martín. Ruta 12, entre 3 de Mayo y El Alcázar, $26^{\circ} 43^{\prime} 01,6 " \mathrm{~S}-54^{\circ} 51^{\prime} 16,8^{\prime}$ ' W, 25-II-2011, fl., Keller \& Paredes 10041 (CTES); San Ignacio, acceso principal a ciudad de San Ignacio, 11-I-2012, fl., Keller \& Paredes 10599 (CTES); Cainguás, ruta prov. 7, $27^{\circ} 03$ ' 32,3” $\mathrm{S}-55^{\circ} 01^{\prime}$ 01,9” W, 11-I2012, fl., Keller \& Paredes 10576 (CTES); idem, Campo Grande, $27^{\circ} 13$ ' 40,8' S - 54 ${ }^{\circ} 58^{\prime} 26,8^{\prime}$ ' W, 11-I-2012, fl., Keller \& Paredes 10583 (CTES).

\section{Agradecimientos}

A los revisores anónimos por sus oportunas sugerencias.

\section{Bibliografía}

BIONDI, D. \& J. H. PEDROSA-MACEDO. 2008. Plantas invasoras encontradas na área urbana de Curitiba (PR). Floresta 38 (1): 129-144.

BIONDO, E., S. T. S. MIOTTO, M. T. SCHIFINOWITTMANN \& B. DECASTRO. 2005. Cytogenetics and cytotaxonomy of Brazilian species of Senna Mill. (Cassieae-Caesalpinioideae-Leguminosae). Caryologia 58 (2): 152-163.

BLUM, C. T., M. BORGO \& A. C. F. SAMPAIO. 2008. Espécies exóticas invasoras na arborização de vias públicas de Maringá-PR. Rev. Soc. Bras. Arb. Urb. 3 (2): 78-97.

BRANCO A., A. C. PINTO, J. SCHRIPSEMA \& R. BRAZ-FILHO. 2011. Anthraquinones from the bark of Senna macranthera. An. Acad. Bras. Ciênc. 83 (4): 1159-1163.
BURKART, A. 1952. Las Leguminosas argentinas, silvestres y cultivadas. 2da. edic. Acme, Buenos Aires. 579 pp.

BURKART, A.1978. Leguminosas. En M. J. Dimitri (ed.), Encicl. Argent. Agricult. Jard. 1: 467-538. 2da. edic. Acme, Buenos Aires.

CADOGAN, L. 1957. Breve contribución al estudio de la nomenclatura guaraní en Botánica. Ministerio de Agricultura y Ganadería. Servicio Técnico Interamericano de Cooperación Agrícola. Boletín nro. 194, Asunción, 49 pp.

CRUZ, C. A. F., H. N. DE PAIVA, J. C. L. NEVES \& A. C. M. C. M. DA CUNHA. 2010. Resposta de mudas de Senna macranthera (DC. ex Collad.) H.S. Irwin \& Barnaby (fedegoso) cultivadas em latossolo vermelhoamarelo distrófico a macronutrientes. Rev. Árvore 34 (1): 13-24.

DEHNEN-SCHMUTZ, K. \& J. TOUZA. 2008. Plant invasions and ornamental horticulture: pathway, propagule pressure and the legal framework. En J. A. Teixeira da Silva (ed.), Floriculture, Ornamental and Plant Biotechnology. Vol. 5: 15-21. Global Sci. Books, London.

DEHNEN-SCHMUTZ, K., J. TOUZA, C. PERRINGS \& M. WILLIAMSON. 2007. The horticultural trade and ornamental plant invasions in Britain. Conservation Biol. 21: 224-231.

DELUCCHI, G. \& J. A. HURRELL. 2011. Taxodium distichum (Cupressaceae) adventicia en la Argentina y Uruguay. Bol. Soc. Argent. Bot. 46 (3-4): 361-367.

DELUCCHI, G. \& H. KELLER. 2010. La naturalización del "níspero", Eriobotrya japonica (Rosaceae, Maloideae), en la Argentina. Bonplandia 19 (1): 71-77.

DELUCCHI, G.; F. BUET COSTANTINO \& E. L. GUERRERO. 2011. Leguminosas exóticas invasoras de la República Argentina: una categorización. Historia Natural (3ra. ser.) 2: 75-84.

DIMITRI, M. J. \& F. RIAL ALBERTI. 1954. Las especies argentinas del género Cassia cultivadas en la Argentina. Revista Invest. Agric. 8 (1): 5-34.

DIAZ, M. A. N., C. C. ROSSI, V. R. MENDONÇA, D. M. SILVA, A. O. B. RIBON, A. P. AGUILAR \& G. D. MUÑOZ. 2010. Screening of medicinal plants for antibacterial activities on Staphylococcus aureus strains isolated from bovine mastitis. Rev. Bras. Farmacogn. 20 (5): 724-728.

GONZÁLES TORRES, D. M. 1996. Catálogo de plantas medicinales (y alimenticias y útiles) usadas en Paraguay. Edit. Comuneros, Asunción. 391 pp.

GUARIZE, L. 2009. Senna macranthera: constituição química e atividades biológicas. Dissertação de Mestrado, Universidade Federal de Juiz de Fora. $122 \mathrm{pp}$.

GUARIZE, L., J. C. DA COSTA, L. B. DUTRA, R. F. MENDES, I. V. LIMA \& E. SCIO. 2011. Antiinflammatory, laxative and intestinal motility effects of Senna macranthera leaves. Nat. Prod. Res. 1: 1-13.

HURRELL, J. A., D. BAZZANO \& G. DELUCCHI. 
2004. Arbustos 2. Nativos y exóticos. En J. A. Hurrell (ed.), Biota Rioplatense IX. Edit. LOLA, Buenos Aires. 288 pp.

HURRELL, J. A., G. DELUCCHI \& J. A. TOLABA. 2010. Presencia de Lilium longiflorum (Liliaceae) adventicia en la Argentina. Bol. Soc. Argent. Bot. 45 (1-2): 195-200.

HURRELL, J. A., P. CABANILLAS \& G. DELUCCHI. 2011a. Wisteria sinensis (Leguminosae) adventicia en la Argentina. Primer registro y mecanismos de expansión. Rev. Mus. Argentino Cienc. Nat., n.s. 13 (2): 125-130.

HURRELL, J. A., G. DELUCCHI \& H. A. KELLER. 2011b. Carya illinoinensis (Juglandaceae) adventicia en la Argentina. Bonplandia 20 (1): 47-54.

HURRELL, J. A., E. A. ULIBARRI, J. P. PUENTES, F. BUET COSTANTINO, P. M. ARENAS, M. L. POCHETTINO. 2011c. Leguminosas medicinales y alimenticias utilizadas en la conurbación Buenos Aires-La Plata, Argentina. Bol. Latinoam. Caribe Plant. Med. Aromat. 10 (5): 443-455.

HURRELL, J. A., E. A. ULIBARRI, P. M. ARENAS, M. L. POCHETTINO. 2011d. Plantas de herboristería. Edit. LOLA, Buenos Aires. 242 pp.

INSTITUTO DE BOTÁNICA DARWINION. 2012. Base de datos. Flora del Cono Sur. Disponible: $<$ http//www2.darwin.edu.ar $>$ [Consulta: 2-II-2012].

IRWIN, H. S. \& R. C. BARNEBY. 1981. Tribe Cassieae. En R. M. Polhill \& P. H. Raven (eds.), Advances in Legume Systematics 1: 97-106. Kew, The Royal Botanic Gardens.

IRWIN, H. S. \& R. C. BARNEBY. 1982. The American Cassiinae. A synoptical revision of Leguminosae, Tribe Cassieae, Subtribe Cassiineae in the New World. Mem. New York Bot. Gard. 35 (2 parts): 1-918.

JARAMILLO-BOTERO, C., R. H. S. SANTOS, M. P. FARDIM, T. M. PONTES \& F. SARMIENTO. 2008. Produção de serapilheira e aporte de nutrientes de espécies arbóreas nativas em um sistema agroflorestal na zona da mata de Minas Gerais. Rev. Árvore 32 (5): 869-877.

JØRGENSEN, P. M. \& S. LEÓN-YÁNEZ (EDS.) 1999. Catalogue of the vascular plants of Ecuador. Monogr. Syst. Bot. Missouri Bot. Gard. 75: 1-1181.

KELLER, H. A., G. DELUCCHI \& H. F. ROMERO. 2011. Camellia sinensis (Theaceae) en la Argentina: naturalización y usos locales. Bol. Soc. Argent. Bot. 46 (1-2): 145-150.

KIILL, L. H. P., F. N. P. HAJI \& P. C. F. LIMA. 2000. Visitantes florais de plantas invasoras de áreas com fruteiras irrigadas. Sci. Agric. 57 (3): 575-580.

LORENZI, H. 1992. Árvores brasileiras: manual de identificação e cultivo de plantas arbóreas nativas do Brasil. Nova Odessa, Editora Plantarum. 352 pp.
MARAZZI, B.; R. H. FORTUNATO, P. K. ENDRESS \& R. SPICHIGER. 2006. Senna (Cassiinae, Leguminosae) in Paraguay: synopsis, occurrence, ecological role and ethnobotany. Candollea 61 (2): 315-329.

MENDONÇA, V. R., L. L. GOMES, G. DIAZ \& M. A. N. DIAZ. 2011. Anthraquinones isolated from Senna macranthera. Nat. Pharm. Technol. 1 (4): 1-3.

MORS, W. B., C. TOLEDO RIZZINI, N. ÁlVARES PEREIRA, R. A. DEFILIPPS. 2000. Medicinal plants of Brazil. Reference Public., New York. 501 pp.

OBERLAENDER, E. R. 2006. Fenologia de Senna macranthera (Collad.) Irwin \& Barneby e Senna multijuga (Rich.) Irwin \& Barneby no Parque Nacional da Serra dos Órgãos e na área urbana de Teresópolis - RJ. Dissertação de Mestrado, Universidade Federal Rural do Rio de Janeiro. 41 pp.

PIRES, L., P. A. J. GORIN, F. REICHER \& M. R. SIERAKOWSKI. 2001. An active heparinoid obtained by suphation of a galactomannan extracted from the endosperm of Senna macranthera seeds. Carboh. Pol. 46 (2): 165-169.

PYSEK, P. \& D. M. RICHARDSON. 2006. The biogeography of naturalization in alien plants. $\mathrm{J}$. Biogeography 12: 2040-2050.

PYŠEK, P., D. M. RICHARDSON, M. REJMÁNEK, G. WEBSTER, M. WILLIAMSON \& J. KIRSCHNER. 2004. Alien plants in checklists and floras: towards better communication between taxonomists and ecologists. Taxon 53 (1): 131-143.

REICHARD, S.H. \& P. WHITE. 2001. Horticulture as a pathway of invasive plant introductions in the United States. Bioscience 51: 103-113.

RICHARDSON, D. M., P. PYŠEK, M. REJMÁNEK, M. G. BARBOUR, F. DANE PANETTA \& C. J. WEST. 2000. Naturalization and invasion of alien plants: concepts and definitions. Diversity Distrib. 6: 93-107.

RODRIGUES, R. S., A. S. FLORES, S. T. S. MIOTTO \& L. R. DE M. BAPTISTA. 2005. O gênero Senna (Leguminosae, Caesalpinioideae) no Rio Grande do Sul, Brasil. Acta Bot. Bras. 19 (1): 1-16.

SOUZA, V. C. \& R. L. C. BORTOLUZZI. 2010. Senna. En R. C. Forzza (coord.), Lista de Espécies da Flora do Brasil. Jardim Botânico do Rio de Janeiro. Disponible: $<$ http://floradobrasil.jbrj.gov. br/2010/FB028201> [Consultado: 1-II-2012].

ULIBARRI, E. A. 2008. Los géneros de Caesalpinioideae (Leguminosae) presentes en Sudamérica. Darwiniana 46 (1): 69-163.

ULIBARRI, E. A, E. GÓMEZ SOSA, A. M. CIALDELLA \& R. H. FORTUNATO. 2006. Leguminosas. Nativas y exóticas. En J. A. Hurrell (ed.), Biota Rioplatense VII: 1-320. Edit. LOLA, Buenos Aires. 